\title{
Ensemble linear regression and Paris' law based methods for structure fatigue crack length estimation and prediction using ultrasonic wave data
}

\author{
Meng Rao ${ }^{1}$, Xingkai Yang ${ }^{1}$, Dongdong Wei ${ }^{1}$, Yuejian Chen ${ }^{1}$, Lijun Meng ${ }^{1,2}$ and Ming J. Zuo ${ }^{1 *}$ \\ ${ }^{1}$ Department of Mechanical Engineering, University of Alberta, Edmonton, T6G 2G8, Canada \\ ${ }^{2}$ School of Electromechanical and Architectural Engineering, Jiang Han University, Wuhan,

$$
\begin{gathered}
\text { 430056, China } \\
\text { *ming.zuo@ualberta.ca }
\end{gathered}
$$

\begin{abstract}
This paper presents the methods developed for the 2019 PHM Conference Data Challenge. The PHM Data Challenge aims to estimate the fatigue crack length of a type of aluminum structure using ultrasonic signals at the current loading cycle, and to predict the crack length at multiple future loading cycles (multiple-step ahead prediction) as accurate as possible. For the crack length estimation, four crack sensitive features are extracted from ultrasonic signals, namely, the first peak value, root mean squared value, log of kurtosis and correlation coefficient. These features and their $2^{\text {nd }}$ order interactions are used as inputs for an ensemble linear regression model which is built to map the features to crack length. The Best Subset Selection method is employed to select optimal features. For the crack length prediction, variations of the Paris' law are derived to describe the mutual relationships between crack length and the number of loading cycles. The specimen material parameters and stress range of the Paris' law are learned using the Genetic Algorithm, and updated with the next-step predicted crack length. The crack length corresponding to a future loading cycle number under constant amplitude cyclic loading and under variable amplitude cyclic loading is predicted respectively. The presented overall methodology achieves a score of 16.14 with the score calculation rule provided by the Data Challenge organization team, and ranks $3^{\text {rd }}$ among all teams.
\end{abstract}

